\title{
La Estrategia Digital de Remed
}

\author{
Marta María Peinador Aguado \\ Estratega Digital y Redes Sociales en REMED
}

\begin{abstract}
Resumen
REMED es la red española de Estrategias Digitales, nacida en abril 2020 durante el confinamiento por la pandemia del Covid19. En ese momento se abrieron las redes sociales de la red, pero faltaba una estrategia digital que sirva de guía para el presente y el futuro de la red y de ejemplo a otros socios que quieran crear la suya. Es un ejercicio de coherencia y transparencia.

Este artículo explica la primera estrategia digital de REMED, desde el punto de partida, pasando por su visión, misión y objetivos y como quiere lograrlos, llegando al público objetivo que hemos seleccionado.

También explica sus canales digitales y sus perfiles en redes sociales como herramientas para llegar a nuestros públicos, comentando su estrategia de contenidos, su evolución, especialmente desde noviembre de 2020, cuando se comenzó a colaborar en el equipo de comunicación y una evaluación de lo conseguido con la creación de la comunidad REMED en redes sociales.
\end{abstract}

Para finalizar con unas conclusiones de cara al futuro de la organización, porque la estrategia digital está en constante replanteamiento, implementación y evaluación.

Palabras clave: Estrategia Digital, Redes Sociales, LínkedIn, Twitter, Facebook, Instagram, REMED

\footnotetext{
Abstract

REMED is the Spanish Network of Digital Strategies, born in April 2020 during confinement due to the Covid19 pandemic. At that time, social networks were opened but a digital strategy was lacking to serve as a guide for the present and the future of the network and as an example to other partners who want to create their own. It is an exercise in coherence and transparency.

This paper explains REMED's first digital strategy, from the starting point, through its vision, mission and objectives and how it was able to achieve them, reaching the target audience that we have selected.

It will also explains its digital channels and its profiles on social networks as tools to reach our audiences, commenting on its content strategy, its evolution, especially since November 2020, when started the collaboration in the
} 
communication team and an evaluation of what was achieved with the creation of the REMED community in social networks was done.

To end, some conclusions for the future of the organization, because the digital strategy is constantly rethinking, implementing and evaluating.

Keywords: Digital Strategy, Social Networks, LinkedIn, Twitter, Facebook, Instagram, REMED 


\section{Introducción}

Remed es la red española de museos y estrategias digitales. Un proyecto que nació en la primavera de 2019, de un grupo de profesores de la Universitat Politècnica de València, y alumnos del Máster Interuniversitario de Gestión Cultural a partir de la alerta sanitaria por la pandemia del Covid19, con la intención de compartir, investigar, documentar y comunicar los procesos de transformación digital que están experimentando los museos en España.

Este proceso había comenzado hace años en algunos museos, pero su implementación se ha convertido en inaplazable para los profesionales desde que la pandemia del Covid19 obligó al cierre de las sedes físicas de los museos.

Sus profesionales tuvieron que trabajar desde sus casas, aprendiendo a marchas forzadas nuevas habilidades digitales si no las tenían (teletrabajo, videoconferencias, presentaciones online en congresos) y a seguir en contacto con los públicos en el mundo digital mediante su web, app, redes sociales y otros proyectos digitales (tours virtuales, conferencias).

En algunos casos los museos abrieron esos perfiles en redes sociales durante el confinamiento, sin pensar en primer lugar una estrategia y en consecuencia escoger en que redes sociales debían estar para llegar a los públicos que querían alcanzar y fidelizar. Y en ocasiones estas tareas las han realizado profesionales sin formación previa en estrategia digital, redes sociales y otras herramientas necesarias para conectar con sus públicos online pero con ganas de explicar el museo a la gente.

Esta transformación digital ya se había implementado con más fuerza en otros países, donde ya tienen eventos que reúnen a los profesionales digitales de los museos plenamente consolidados (Museums and the Web y Museums Computer Netwotk en EEUU, Museum Connections en Francia, Museums Computer Group en Gran Bretaña, Be Museum in Bélgica).

Este documento quiere ser un plan para que Remed se convierta en el referente de los profesionales del sector digital en los museos en España e Hispanoamérica y explicar cómo su web y sus redes sociales le ayudarán a conseguirlo.

\section{Misión, Visión y Objetivos}

Mientras en el extranjero hace años que se celebran congresos y eventos para hablar de la transformación digital en los museos, en España faltaba crear una red para reunir a todos los profesionales de museos, instituciones, empresas y centros de investigación en España e Hispanoamérica, interesados en este tema y un evento para poner en común sus ideas y proyectos.

Hagamos un análisis DAFO de la situación de partida al emprender el proyecto: 
Las debilidades de REMED son ser un proyecto poco conocido y la falta de fuentes de financiación para seguir creciendo.

La amenaza es la crisis económica que evita las inversiones en la digitalización de los museos, el reciclaje de los profesionales y la ampliación de equipos con profesionales formados cuando hay una demanda de actividad digital en el sector.

La fortaleza del proyecto es un equipo comprometido y un proyecto participativo que ha logrado atraer a 700 profesionales, que dan fuerza a la red y les conciencia de la importancia de la digitalización en los museos para funcionar de una manera más eficiente y coordinada y llegar a nuevos públicos.

La oportunidad de REMED es llenar el vacío existente hasta ahora de no tener un foro de debate, intercambio de ideas y formación y un evento que sea punto de encuentro para los profesionales que trabajan en entornos digitales y a los que quieran iniciarse en el sector.

Por eso REMED tiene los siguientes objetivos:

- Reunir a los profesionales que trabajen en proyectos de transformación digital en los museos y el patrimonio.

- Formar a los profesionales de museos en habilidades digitales

- Ser un foro de intercambio de ideas y experiencias de los profesionales.

- Documentar los procesos de transformación digital de los museos

La misión de REMED es ser un foro para el intercambio de ideas y experiencias sobre la transformación digital en los museos, reuniendo a sus profesionales, formándolos y documentando sus proyectos.

REMED se divide en 4 áreas de trabajo:

- Coordinación (toma de decisiones)

- Comunicación (Estrategia Digital, Redes Sociales y Relaciones Externas)

- Investigación ( Documentación de procesos de transformación digital)

- Profesionales de Museos (Estudio del sector)

Las Redes Sociales serán claves para cumplir nuestra misión y objetivos:

- Incrementarán la visibilidad de REMED. Compartiremos noticias, eventos y proyectos que atraigan a profesionales de museos en el ámbito digital.

- Nos convertirán en líderes en el sector en el ámbito hispanohablante. Seremos la referencia en información sobre museos y mundo digital.

- Nos ayudarán a crear y mantener alianzas con entidades similares, que organicen eventos sobre museos y estrategias digitales y conseguir sponsors para nuestros proyectos. 
- Promoverán el desarrollo profesional de los socios, compartiendo noticias que inspiren y sus proyectos y difundiendo las actividades que organizamos en REMED.

\section{Desarrollo de la Innovación}

\subsection{Cómo queremos lograr nuestros objetivos}

- Dando a conocer REMED en las redes sociales, los medios de comunicación y eventos relacionados con la transformación digital en los museos, para hacer crecer la red de profesionales.

- Organizando el congreso anual CIMED (Universitat Politécnica de Valencia , 2020), el primer congreso en España de Museos y Estrategias Digitales, que muestre los mejores proyectos de los profesionales del sector digital en los museos que sirvan de inspiración para crear otros nuevos.

- Organizando actividades formativas durante todo el año, talleres prácticos que hagan más fácil la transformación digital para las personas que se han de iniciar o mejorar sus proyectos digitales.

- Siendo un foro permanente debate e intercambio de ideas y experiencias entre los/as profesionales, con actividades como el \#ViernesREMED. (Universitat Politécnica de Valencia , 2020)

- Compartiendo noticias, eventos, proyectos de investigación e informes sobre procesos de transformación digital de los museos

\section{2 ¿Quien es nuestro público objetivo?}

- Profesionales de museos que trabajen en el área digital: web, estrategia digital, redes sociales y proyectos digitales (apps, VR, AR).

- Profesionales de museos que quieran formarse en habilidades digitales para emprender proyectos de este tipo.

- Personal universitario involucrado en proyectos de investigación sobre digitalización en los museos.

- Empresas que hagan proyectos digitales para museos y patrimonio.

- Agencias de comunicación digital que trabajan para museos 


\section{Estrategia de contenidos}

Un buen contenido en nuestras redes será la mejor herramienta para llegar a nuestros públicos, coherente con nuestros objetivos, planificando, creando y evaluando nuestras publicaciones y su éxito.

1. Contenido propio: noticias sobre el congreso CIMED 2021, los debates del Viernes REMED, actividades formativas.

2. Contenido de nuestros socios: compartir y comentar sus campañas en RRSS, informes, iniciativas.

3. Contenido curado: noticias en prensa, radio y TV sobre transformación digital en los museos

4. Eventos de museos y mundo digital, tanto nacionales como internacionales.

\section{Herramientas y canales digitales}

REMED cuenta con varios recursos digitales para darse a conocer, formar a profesionales, debatir y compartir información y experiencias.

- La página web https://remed.webs.upv.es/

- La newsletter, para los socios de la red

- el canal de Microsoft teams para las reuniones y debates

- 4 canales de redes sociales: LinkedIn, Facebook, Twitter e Instagram.

- Un canal de Youtube donde se han colgado videos de los Viernes Remed (Canal Youtube REMED)

En el último trimestre, la comunidad de seguidores ha crecido, especialmente en LinkedIn, Twitter e Instagram.

\begin{tabular}{|l|l|l|l|l|}
\hline \multicolumn{5}{|c|}{$\begin{array}{l}\text { EVOLUCIÓN DE LOS SEGUIDORES } \\
\text { EN REDES SOCIALES }\end{array}$} \\
\hline MES & FACEBOOK & TWITTER & INSTAGRAM & LINKEDIN \\
\hline $\begin{array}{l}\text { Noviembre } \\
2020\end{array}$ & 334 & 165 & 178 & 38 \\
\hline $\begin{array}{l}\text { Diciembre } \\
2020\end{array}$ & 366 & 245 & 225 & 300 \\
\hline Enero 2020 & 466 & 390 & 228 & 362 \\
\hline
\end{tabular}




\begin{tabular}{|l|l|l|l|l|}
\hline 1 Marzo 2020 & 555 & 586 & 525 & 733 \\
\hline
\end{tabular}

Fig. 1. Evolución de los seguidores de REMED en Redes Sociales

\section{Resultados}

6.1 Twitter@remed 20 https://twitter.com/remed_20

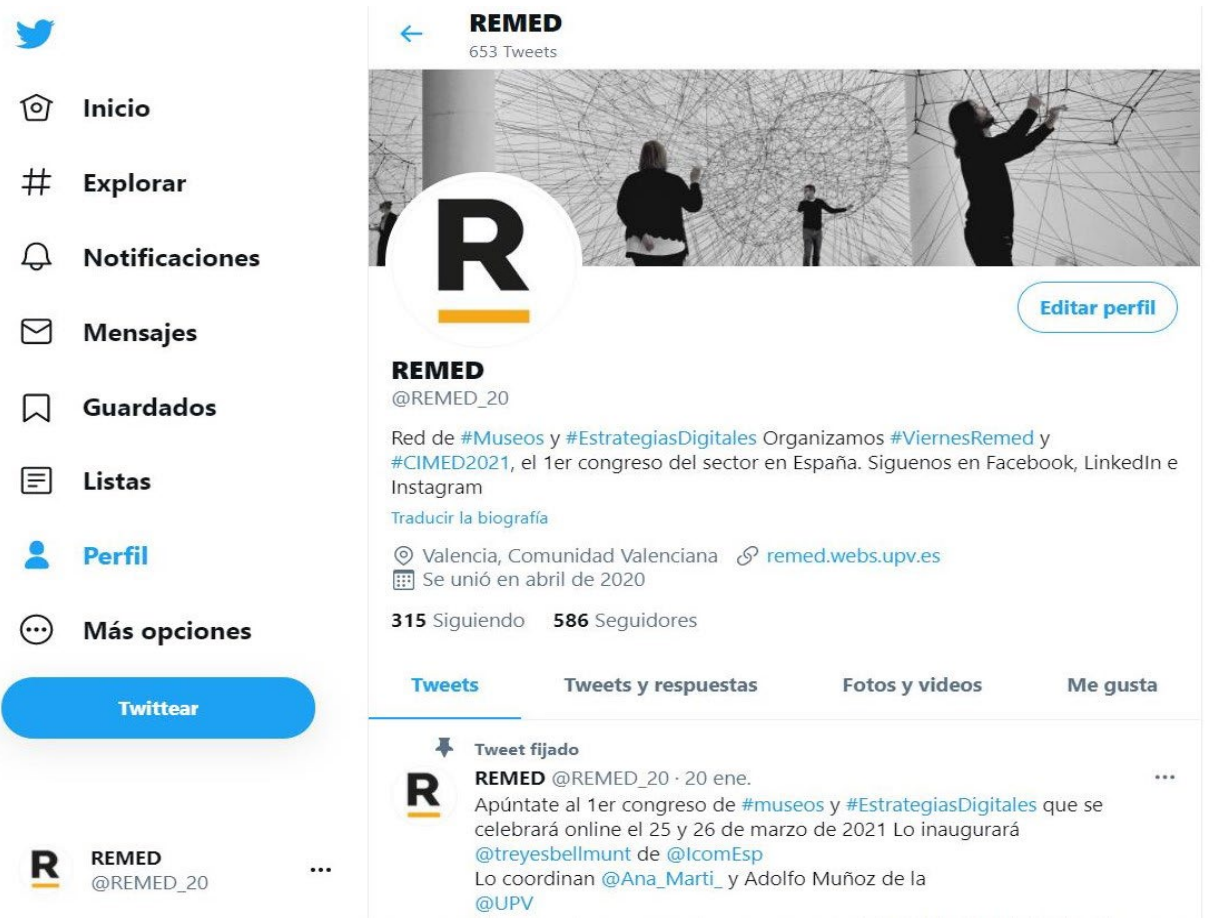

Fig. 2 Perfil de Twitter de REMED

\begin{tabular}{|l|l|l|l|l|l|}
\hline $\begin{array}{l}\text { Estadísticas } \\
\text { en Twitter }\end{array}$ & $\begin{array}{l}\text { Nuevos } \\
\text { seguidores }\end{array}$ & $\begin{array}{l}\text { Visitas al } \\
\text { perfil }\end{array}$ & Impresiones & Menciones & $\begin{array}{l}\text { Número } \\
\text { de tuits }\end{array}$ \\
\hline $\begin{array}{l}\text { Octubre } \\
2020\end{array}$ & 34 & 196 & 4517 & 9 & 7 \\
\hline
\end{tabular}




\begin{tabular}{|l|l|l|l|l|l|}
\hline $\begin{array}{l}\text { Noviembre } \\
2020\end{array}$ & 56 & 891 & $26,5 \mathrm{mil}$ & 24 & 72 \\
\hline $\begin{array}{l}\text { Diciembre } \\
2020\end{array}$ & 101 & 3140 & $75,7 \mathrm{mil}$ & 77 & 118 \\
\hline Enero 2021 & 80 & 2234 & $96,1 \mathrm{mil}$ & 34 & 50 \\
\hline $\begin{array}{l}\text { Febrero } \\
2021\end{array}$ & 110 & 5154 & $69,6 \mathrm{mil}$ & 69 & 139 \\
\hline
\end{tabular}

Fig. 3 Estadísticas del Twitter de Remed (octubre 2020 - febrero (Analytics cuenta de Twitter de REMED)

6.2 Linkedin (Pagina de Linkedin de REMED) https://www.linkedin.com/company/54162324/admin/

in $\mathbf{Q}_{\text {Buscar }}$
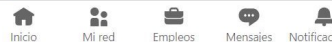

R REMED - Red de Museos y Estrategias Digitales Vista de superadministrador

Inicio Contenido Analisis - Actividad 1
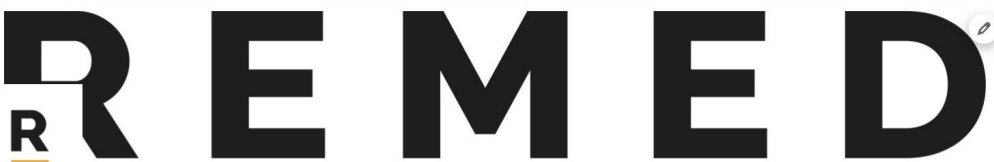

REMED - Red de Museos y Estrategias Digit..

Plataforma abierta para consultar compartir e investigar las estrategias digitales de los museos durante el COVID-19:

Museos e instituciones - Valencia 734 seguidores

Fig. 4 Perfil de LinkedIn de REMED 


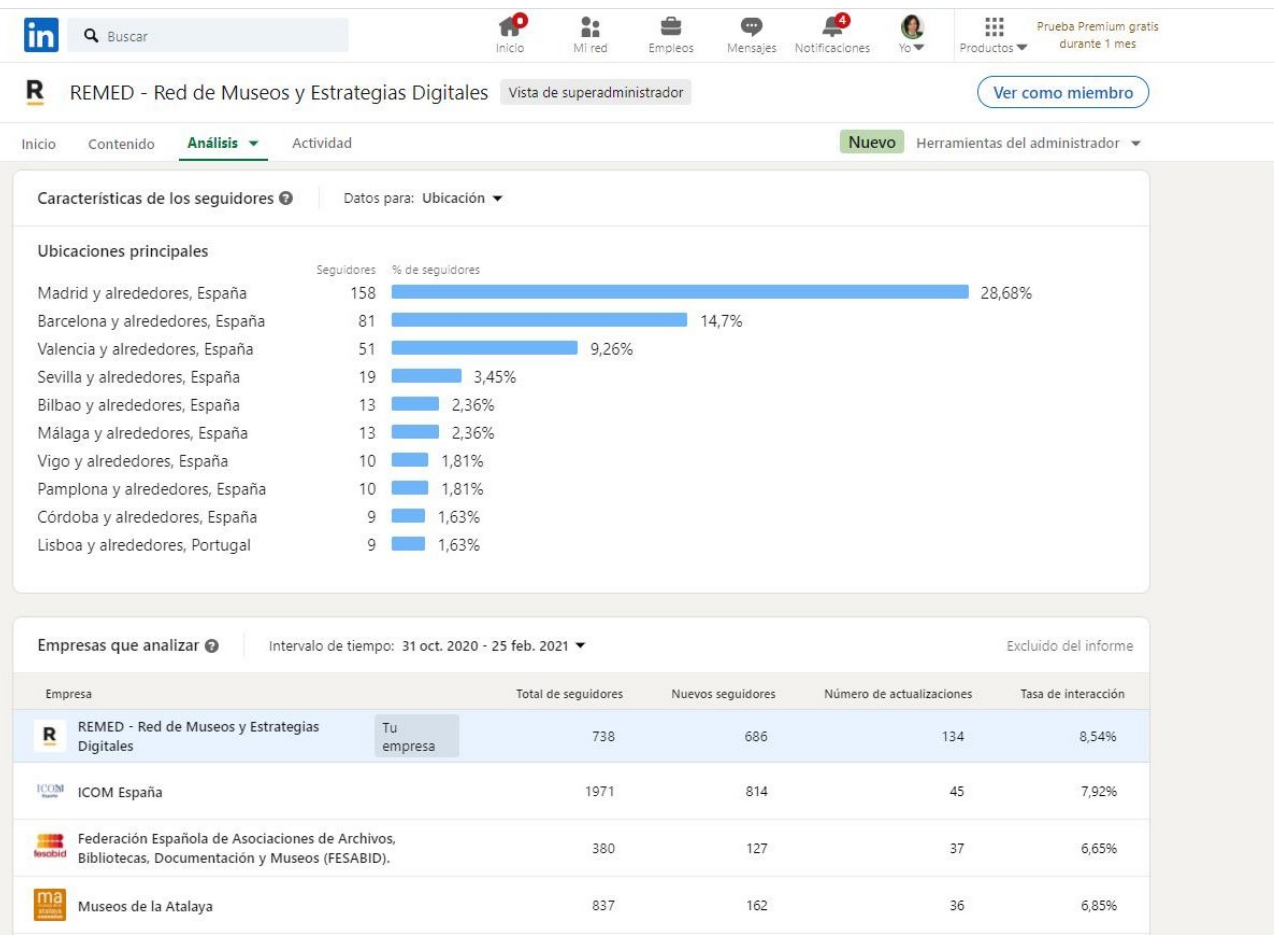

Fig.5. Estadísticas del LinkedIn de Remed

6.3 Facebook (Facebook REMED) https://www.facebook.com/REMED$\underline{104505524572706 /}$ 


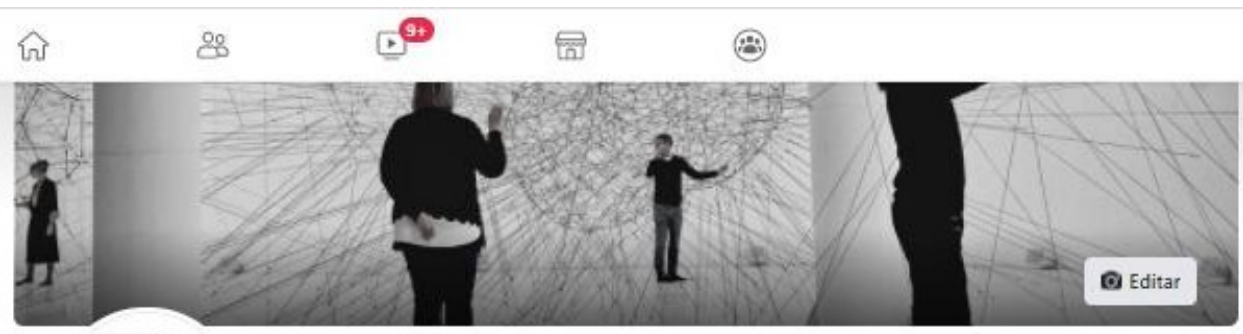

\section{REMED}

@remed20 - Sitio web de sociedad y cultura Editar Contactar

\begin{tabular}{ll|l|l|l|l}
\hline Inicio Información Ver más & $\$$ Promocionar & $\odot$ Ver como visitante & Q & $\ldots$ \\
\hline
\end{tabular}

(9) Administra tu página y cuenta de Instagram juntas con Business Suite.

Crear anuncio Ver todo

¿Cómo quieres hacer crecer tu empresa?

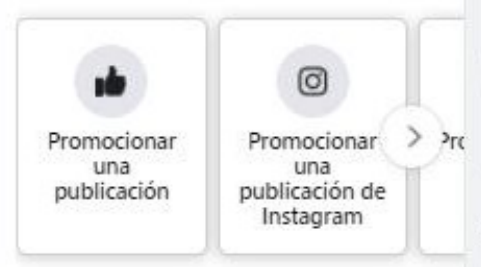

Anuncios automatizados

Obtein anuncios personalizadas pue se ajuster con el tiempo pera canseguir mejores resultados.

\section{Herramientas gratuitas para empresas de Facebook}

Vender tus productos
Da a conocer tus productos entre
millones de clientes potenciales en..
Añadir reservas de citas
Mostrar tus servicios y
disponibilidad para que las person...
Mostrar tu menú
Permite que las personas exploren
tu menú y hagan pedidos en..

Estadísticas
Últimos 28 dias : 19 dic - 15 ene-
Personas alcanzadas $\quad 329,677$

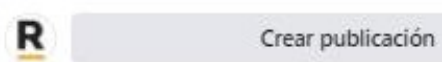

Crear Foto/video
Cren directo $\quad$ Evento
PUBLICACIÓN FUADA

R REMED

El 25 y 26 de marzo de 2021 se celebrará el 1er Congreso Internacional de Museos y Estrategias Digitales, \#CIMED2021. organizado por la Universitat Politècnica de València UPV, en colaboración con REMED

Esperamos recibir tu comunicación. Puedes enviárnosla hasta el 8 de enero de 2021. Consulta en el link como prepararla. https://remed.webs.upv.es/.../convocatoria-de-comunicaciones Las actas del congreso se publicarán indexadas con ISBN y DOL. ¡Te esperamos! _ Ver más

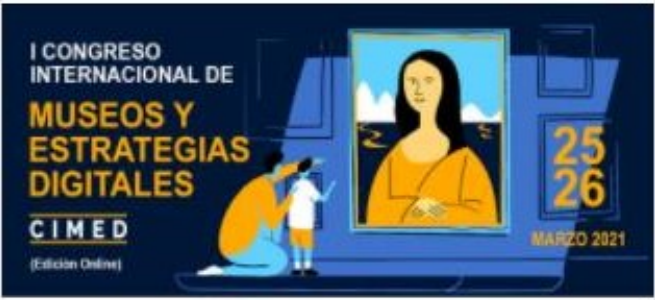

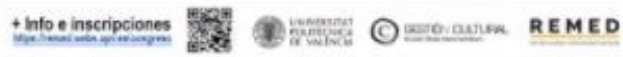
\begin{tabular}{lll}
9256 & 496 & Promocionar publicación \\
\hline
\end{tabular} Q0 231 comentario 66 veces compartida

Fig. 6 Perfil de Facebook de Remed 


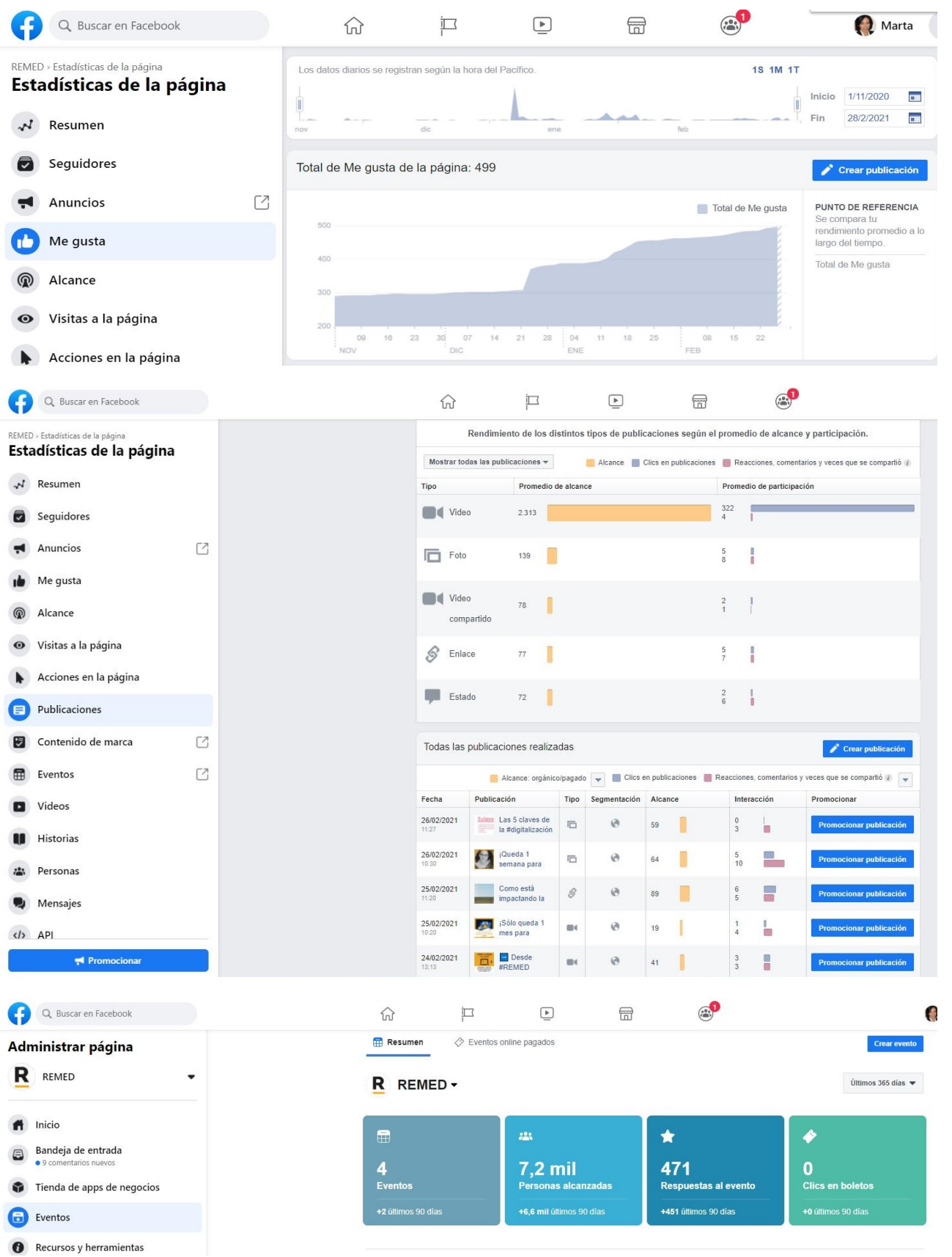

Fig. 7 Estadísticas del perfil de facebook de Remed 
6.4 Instagram @remed_20 (Cuenta de Instagram de REMED) https://www.instagram.com/remed_20/

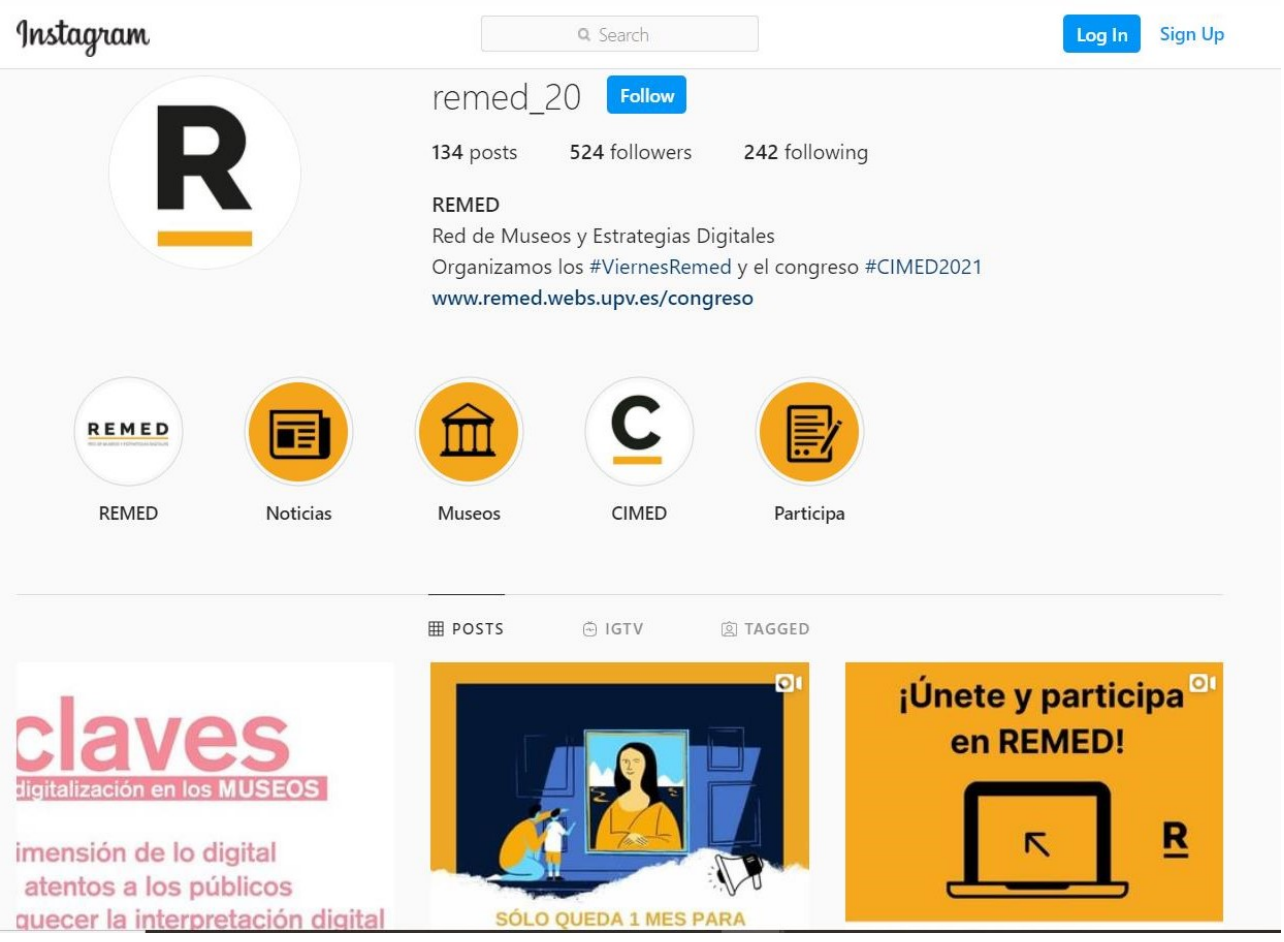

Fig. 8 Perfil de Instagram de Remed 


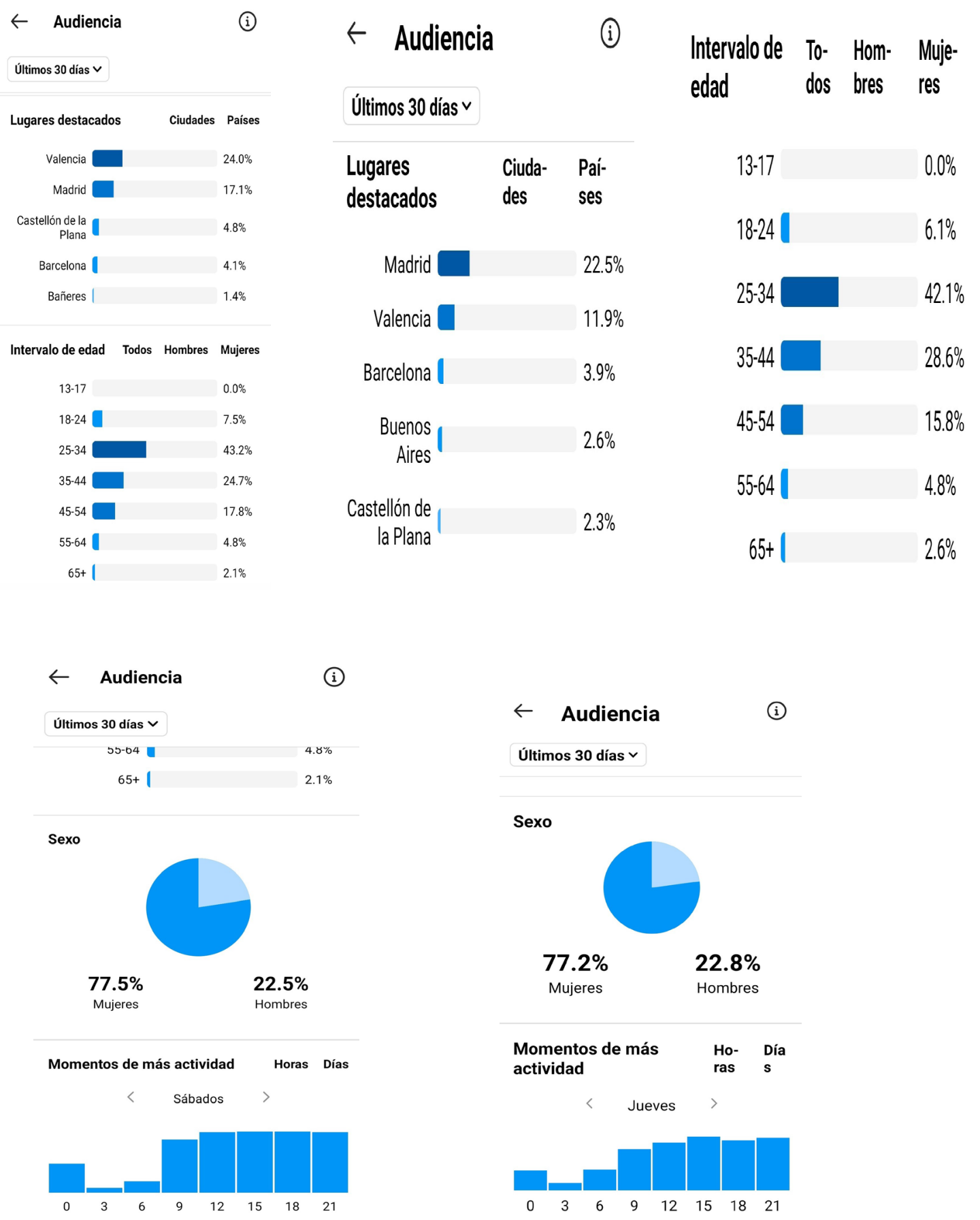

Fig. 9 Estadísticas Instagram de Remed (Enero y Febrero de 2021) 


\subsection{La web de remed}

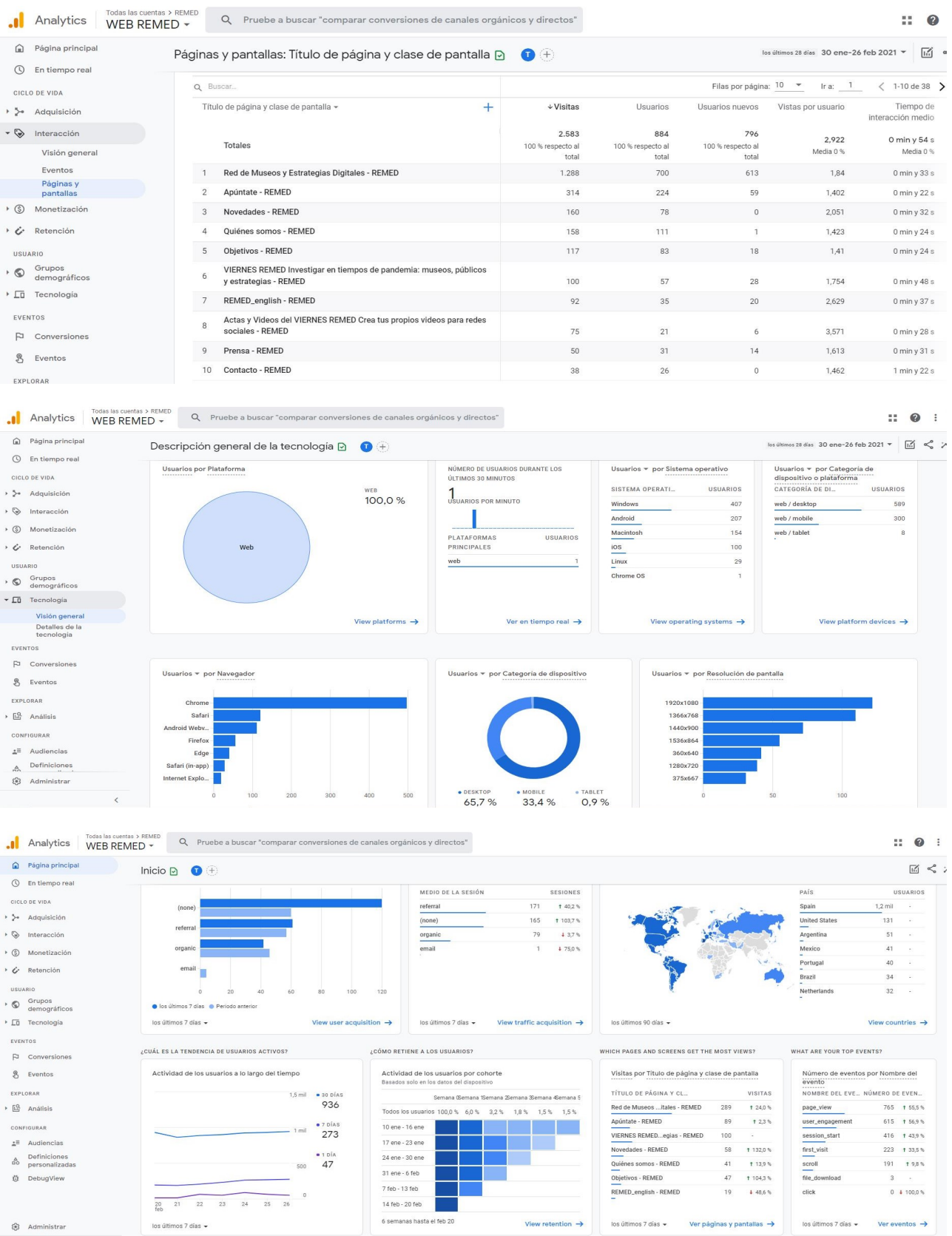

Fig.10 Estadísticas de la web de Remed en Google Analytics

La mayoría de los visitantes a la web de REMED son de España, seguidos a mucha distancia por Estados Unidos, Argentina, México, Portugal y Brasil. 
Los visitantes internacionales pueden venir de haber hablado de REMED en Congresos Internacionales (MCN y MuseumNext) y también hay consultas al apartado de la web traducido al inglés.

Las páginas más visitadas del sitio son las de inicio e inscripción en la Red REMED. El 65\% consulta la web desde el ordenador, el 33\% desde el móvil y sólo el 0,9\% desde una Tablet. El navegador más utilizado es Chrome y los sistema operativos, Windows en el ordenador y Android en el móvil.

Twitter es la red donde se producen más interacciones y comentarios y en tres meses hemos triplicado el número de seguidores, algunos de los cuales son auténticos prescriptores de la red. Sólo en febrero hemos tenido más de 100 nuevos seguidores.

LinkedIn es la red donde se ha producido el crecimiento más espectacular, de 38 a 734 seguidores en 4 meses. El 28\% de los seguidores son de Madrid, el 14,7\% de Barcelona y el 9,2\% de Valencia. El resto son de otros lugares de España y sólo el 1,6\% son de Portugal.

En ambas redes, los ponentes han contribuido a la difusión del congreso CIMED 2021, compartiendo nuestras publicaciones o haciendo las suyas propias que también hemos compartido.

Facebook es la red donde el crecimiento ha sido más moderado, pero la publicación que anuncia el congreso, fijada en el perfil se ha compartido 70 veces y ha alcanzado a 9977 personas. Las publicaciones sobre los Viernes REMED también despiertan mucho interés así como las que tienen diseños animados realizados en Canva. El número de me gustas a la página no ha dejado de crecer.

Instagram es usada mayoritariamente por mujeres (75\%) entre 25 y 44 años (42\%) de Madrid, Valencia y Barcelona. Se utiliza para hacer consultas y preguntas que se contestan rápidamente.

\section{Conclusiones}

La tarea de aumentar y mejorar la comunidad de REMED no ha sido fácil, pero se ha consegido mejorar gracias a la implantación de una estrategia digital y de contenidos, pasión por el proyecto y trabajo duro. Además de trabajar otros aspectos igualmente relevantes, como:

- Crear y curar contenido, interactuar con la comunidad y analizar el resultado de las acciones exige mucho tiempo pero es apasionante.

- Dedicar más tiempo a seleccionar información, diseñar en Canva (CANVA) o pensar en las publicaciones, que a publicar. 
- Hay que seguir avanzando, evaluando todo lo hecho hasta ahora y buscar nuevos retos.

Retos de futuro:

- Consolidar la red y hacerla crecer tanto en España como en Hispanoamérica.

- Conocer más a fondo a los profesionales de museos y sus necesidades

- Continuar con las actividades formativas durante todo el año, entre congreso y congreso CIMED.

- Fomentar la participación activa de los miembros de la Red

- Conseguir financiación para organizar un congreso aún mejor y actividades formativas adecuadas a las necesidades de los socios/as.

- Seguir investigando la transformación digital de los museos.

Gracias a Natalia Almudí y Belen Perelló y Rubén David Costa por la ayuda en los diseños en Canva y colaborar en publicaciones en las redes sociales.

\section{Referencias}

CCCB dossier. Estrategia Digital para museos http://lab.cccb.org/en/dossier/digitalstrategies-at-museums/. CCCBLab.

EVJEN, MAX; STEIN, ROBERT; ALLEN-GREIL, DANA; LYTLE-PAINTER, EMILY. STRATEGY 3.0: What Is Digital Strategy Now?. Congreso Museums and the Web 2017. $<\quad$ https://mw17.mwconf.org/proposal/strategy-3-0-what-is-digital-strategy-now-2/> (consulta febrero 2021)

MUÑOZ, GEMMA; ELÓSEGUI, TRISTÁN. El arte de medir Manual de Analítica Web. Profit Editorial. Barcelona, 2011

MORRISON, ALEX. Cogapp. Digital Strategy for museums https://www.cogapp.com/digital-strategy (consulta Febrero 2021)

RODÀ, CONXA; ROCA, GENIS ; SIERRA, ALBERT Y OTROS. Curso Estrategia Digital en los Museos. Museu Nacional d'Art de Catalunya. 15 horas. Barcelona, 2015. https://www.museunacional.cat/es/actividades/estrategia-digital-en-los-museos (consulta febrero 2021)

RODÀ CONXA; MERÍN, CLARA, GÓMEZ, SOLEDAD; VILLAESPESA, ELENA; TASICH, TIJANA. Curso de Especialización Estrategia Digital en Organizaciones 
Culturales organizado por la UOC y el Museu Nacional d'Art de Catalunya. Octubre 2016 - $\quad$ Febrero 2017. https:/estudios.uoc.edu/es/masters-posgradosespecializaciones/especializacion/artes-humanidades/estrategia-digital-organizacionesculturales-mnac/presentacio. (consulta febrero 2021)

ROJAS, PEDRO (2011). Community management en una semana. Gestión 2000, Grupo Planeta. Barcelona, 2011

RODRIGUEZ FERNÁNDEZ, OSCAR. Curso de Community Manager. Edición 2016. Anaya Multimedia. Madrid, 2015.

STEELLANDT, KATRIEN, Implementing a Digital Strategy for Musea Brugge: from goals to principles. Congreso Museums and the Web 2020. https://mw20.museweb.net/paper/implementing-a-digital-strategy-for-musea-bruggefrom-goals-to-principles/ (Consulta febrero 2021) 